\title{
La Acción Evaluativa en Profesores de Educación Física: Una Investigación Multi-Casos
}

\section{Assessment Action of Physical Education Teachers: A Multi- Case Study}

\author{
Beatriz Elena Chaverra Fernández *1 \\ Juan Luis Hernández Álvarez ${ }^{2}$ \\ ${ }^{1}$ Universidad de Antioquia, Colombia \\ ${ }^{2}$ Universidad Autónoma de Madrid, España
}

\begin{abstract}
La acción evaluativa se asume como todas las actividades que realiza el docente en la fase interactiva de la enseñanza, que están enfocadas en la evaluación y reflejan su pensamiento práctico. Si bien se entiende que esa acción evaluativa constituye una globalidad, analizar cada uno de los elementos que componen la evaluación (momentos, actores, instrumentos y contenidos) permite identificar mejor las tramas y relaciones de esta acción global. El objetivo del estudio fue analizar la acción evaluativa durante una unidad didáctica de un grupo de profesores de educación física de educación secundaria y media de Medellín (Colombia). La investigación se realizó mediante un diseño cualitativo, específicamente con un enfoque metodológico de estudio de caso múltiple. La recolección de la información se realizó por medio de la observación a través de la grabación en audio y video de todas las sesiones de clase de cada docente (51 en total) y el análisis documental. Entre los principales hallazgos se encuentran que la tradición sumativa y acreditadora de la evaluación influye fuertemente en la acción evaluativa del profesorado. Esta postura se ve reflejada en la recolección de información asistemática, la carencia de instrumentos y criterios concretos y, especialmente, en el protagonismo otorgando a la calificación y cómo ella permea todo el proceso evaluativo.
\end{abstract}

Palabras clave: Evaluación del estudiante; Método de evaluación; Enseñanza secundaria; Educación física; Investigación pedagógica.

\begin{abstract}
The assessment action is assumed as all the activities carried out by the teacher in the interactive phase of teaching, focused on the assessment and reflect the practical thinking of teacher. Although it is understood that this assessment action constitutes a totality, analyzing each of the elements that makeup the assessment (moments, actors, instruments and contents) allows to identify the plots and relationships of a better way. The objective of this study was to analyze the assessment action during a didactic unit of a group of Physical Education teachers of middle and high school of Medellin (Colombia). The research was conducted through a qualitative design, specifically with a methodological approach of multiple case study. The information was collected through observation using audio and video recording of all class sessions of every teacher (a total of) and documentary analysis. Among the main findings are that the summative and accrediting tradition of the assessment strongly influences the assessment action of the teachers. This position is reflected in the collection of unsystematic information, the lack of specific instruments and criteria, especially, in the leading role in awarding the grade and how it permeates the entire assessment process.
\end{abstract}

Keyword: Student evaluation; Assessment methods; Secondary education; Physical education; Pedagogical research.

*Contacto: beatriz.chaverra@udea.edu.co

issn: 1989-0397

www.rinace.net/riee/

https://revistas.uam.es/riee
Recibido: $\quad 16$ de enero de 2019

$1^{\text {a }}$ Evaluación: 26 de febrero de 2019

$2^{\text {a }}$ Evaluación: 21 de marzo de 2019

Aceptado: $\quad$ 04 de abril de 2019 


\section{Introducción}

En la evaluación confluyen múltiples miradas, funciones, procesos y concepciones, de forma que lleva a diferentes autores a considerarla como el aspecto más complejo de la educación (Cabezas, González y Carpintero, 2009; Cerda, 2000; Rosales, 2000) y, en algunos casos, a describirla bien como una auténtica barrera o como un facilitador de reformas educativas, en función de si es o no posible transformar las prácticas evaluativas que se llevan a cabo (Nunziati, 1990).

En la actualidad, la evaluación ha cobrado gran relevancia en el entorno educativo por su función social en el modelo económico vigente, de ahí que sea uno de los elementos educativos que más atención acapara de la sociedad por las funciones que le han atribuido para medir, clasificar y estandarizar el conocimiento. Sin embargo, desde una mirada pedagógica, la evaluación no sólo cumple con una función acreditativa, sino formativa, como una fuente de aprendizaje para los actores educativos (Barrientos y López-Pastor, 2017; Carless, 2007).

La evaluación en educación física, como área educativa, no está exenta de estas contradicciones. Son múltiples los estudios y avances teóricos que apuestan por el valor de la evaluación en el área desde un enfoque crítico y reflexivo, desde allí se promueve la participación activa del estudiantado y el profesorado y se cuestionan las prácticas tradicionales escasamente formativas (Benevides y Santos, 2004; Conte y Moreno, 2000; López-Pastor, 2005; Ní Chróinín y Cosgrave, 2013; Ureña, Ureña, Velandrino y Alarcón, 2006). Sin embargo, surgen nuevos interrogantes que amplían el marco de investigación en la búsqueda de una educación física de calidad a partir del análisis y la transformación de las prácticas evaluativas.

Ahondar en cómo se lleva a cabo la evaluación, puede suponer un avance en el conocimiento teórico y/o práctico sobre la calidad de la enseñanza en educación física, específicamente desde las prácticas evaluativas. Entendida la calidad, en los términos de Velázquez et al. (2007), como una intervención docente que:

\section{... fomente la autonomía, el sentido crítico y participativo, la claridad intelectual, el bienestar personal, la responsabilidad, y el gusto por el saber y por el saber hacer, promoviendo, por tanto, la adquisición y el desarrollo de aquellos conocimientos teóricos y prácticos, actitudes y capacidades que hacen posible a su vez el desarrollo personal y social del alumnado, de manera coherente con los requerimientos y necesidades de las sociedades democráticas. (p. 451)}

Para López, Barba, Monjas, Manrique, Heras y Gómez (2007), la evaluación debe tener como finalidad principal mejorar los procesos de enseñanza - aprendizaje "tanto en lo relativo al aprendizaje del alumnado como a la mejora de la práctica docente” (p. 73). En consecuencia, un adecuado proceso evaluativo aporta elementos claves para conseguir una educación física de calidad.

De acuerdo a esta intención formativa de la evaluación, este estudio se centró en analizar cómo actúa un grupo de docentes de educación física sobre la evaluación en la fase interactiva de la enseñanza propuesta por Jackson (1992). Numerosos estudios se refieren a la acción interactiva del profesorado en el proceso de enseñanza y aprendizaje, con el objetivo de descifrar la complejidad de sus comportamientos y discursos (Del Villar, Ramos, Cervelló, Julián y Jiménez, 2002; Fernández, 2007; Giraldo, Rubio y Fernández, 2009; Pradas, 2010; Tristán, 2010; Velázquez et al., 2007). No obstante, salvo algunas 
orientaciones sobre el denominado feedback evaluativo (López-Rodríguez, 2012), los estudios no han centrado la mirada en la evaluación o lo han hecho de manera superficial.

\section{Revisión de la literatura}

Las investigaciones sobre el pensamiento del profesor han intentado descubrir lo que el profesorado piensa mientras interactúa con el estudiantado, buscando establecer en qué medida se toman decisiones en la acción educativa que no estaban contempladas en la planificación y a la vez, si esas decisiones modifican la planificación siguiente y la conducta (Clark y Peterson, 1990; Hall y Smith, 2006).

Desde un enfoque cognitivo, los primeros estudios conformaron dos líneas de investigación que pretendían generar conocimiento sobre las decisiones interactivas del profesorado; una enfocada hacia el procesamiento de la información y otra hacia la toma de decisiones. No obstante, los resultados de los estudios dejaron en evidencia procesos cognitivos comunes en ambas líneas, así lo confirman Pérez y Gimeno (1988):

Cuando se estudian los procesos mentales de la fase interactiva de la enseñanza se considera al profesor como un profesional que continuamente toma decisiones, comprobando las características de la situación y sus cambios, procesando la información que recibe del contexto, decidiendo los próximos pasos de cada intervención, orientando la acción en función de las decisiones tomadas y observando y valorando el efecto de aquellas actuaciones sobre los alumnos. (p. 41)

Esta visión desde el enfoque cognitivo, centrado exclusivamente en los procesos mentales del profesorado, pretende ser superada por una mirada más pedagógica que involucra los contenidos, la escuela, la cultura, el contexto..., otros elementos que influyen en la enseñanza y el aprendizaje. En este sentido, continúan teniendo vigencia los denominados modelos alternativos que proponen Pérez y Gimeno desde 1988, donde resaltan la importancia de "respetar la complejidad del pensamiento que realmente influye en la actuación del profesor, para que sobre su análisis y valoración reflexiva se articule, contraste, se estime su potencialidad y, en su caso, se modifique o reformule” (1988, p. 55).

Se propone desde este enfoque, una enseñanza reflexiva en la que el profesorado no sólo analice cómo aplica en sus clases las teorías generadas en otros lugares, sino que critique y desarrolle sus propias teorías y prácticas, reflexionando en la acción y sobre ella (Shön, 1998; Zeichner, 1993).

Esta visión del maestro reflexivo, es reflejo de una manera de asumir la investigación del pensamiento del profesor desde la compleja relación entre cognición y acción, es decir, conocer tanto sus teorías implícitas (ideas, creencias, concepciones) como el conocimiento práctico que se genera en la incertidumbre del encuentro educativo, tanto en profesores noveles como expertos (García, 2003; Jinhong, 2012). En consecuencia, se asume el pensamiento práctico del profesor como una mezcla de teorías formales y conocimientos construidos a partir de la experiencia profesional, asimilado de la tradición y la cultura profesional y extra profesional. Como lo plantea Pradas (2010), indagar sobre el conocimiento práctico requiere analizar lo que hacen y piensan los docentes cuando están en la clase, en su práctica educativa.

Cómo se mencionó anteriormente, Jackson (1992) sugiere analizar el proceso de enseñanza en tres fases (pre-activa, interactiva y postactiva). La fase interactiva, donde 
centramos nuestro estudio, se considera el momento principal del proceso educativo, debido a que es allí donde se presenta el encuentro, el intercambio, la comunicación; donde el docente tiene la oportunidad de incidir de manera directa sobre el aprendizaje del estudiantado, donde aplica lo que ha planificado y, a la vez, se enfrenta a situaciones que no estaban previstas, en definitiva, es un momento en que se toman numerosas decisiones con relación a su acción y a la relación con el estudiantado (Giné, Parcerisa, Llena, París y Quinquer, 2003).

Conocer los factores que influyen en las decisiones y pensamientos del profesorado en la fase interactiva y en qué medida se modifican sus conductas, requiere preguntarse por el comportamiento del profesorado. Especialmente sobre la evaluación caben preguntas como: ¿Qué procedimientos evaluativos utiliza el profesorado? ¿Cuál es la participación del estudiantado en la evaluación? ¿Cómo plantean las tareas evaluativas y cómo reacciona ante ellas el estudiantado? ¿Qué contenidos se evalúan en la clase? ¿Cómo se evalúa la enseñanza?, etc.

En este punto, es necesario precisar qué es la acción evaluativa en este estudio y así, focalizar la interpretación de la información. Asumimos la acción evaluativa como aquellas prácticas que realiza el docente en su clase que están enfocadas hacia la evaluación, las cuales manifiestan su pensamiento práctico y permiten comprender cómo se realiza la evaluación clase a clase, qué estrategias e instrumentos utilizan, cómo participa el estudiantado, qué papel tiene la calificación, en qué momento se evalúa, etc., (ChaverraFernández, 2017a). Esta acción también se acompaña de un discurso evaluativo, sin embargo, no será motivo de análisis en este artículo (Chaverra-Fernández, 2017b).

Por todo lo anterior, el objetivo de esta investigación fue analizar la acción evaluativa del profesorado durante una unidad didáctica, es decir, cómo se desarrolla la evaluación clase a clase, qué estrategias e instrumentos utilizan, cómo participa el estudiantado, cómo surge la calificación, en qué momento de la unidad didáctica evalúan, etc.

\section{Método}

\subsection{Enfoque metodológico}

Esta investigación se llevó a cabo a través de la metodología cualitativa, la cual, como actividad situada, ubica al observador en el mundo particular de los participantes (Denzin y Lincoln, 2005). Es una interpretación naturalista de las realidades, lo que significa que las cosas se estudian en su forma o contexto natural, tratando de entender, interpretar y comprender el fenómeno en términos de lo que significan para los sujetos.

Específicamente se abordó el estudio de caso múltiple (Stake, 2010) y se respetaron los lineamientos del autor, quien advierte que los casos individuales deben ser estudiados para aprender acerca de su complejidad y han de entenderse cada uno en profundidad. Luego de analizarlos individualmente, se retomó la recomendación de Tójar y Mena (2011) de revisar las convergencias y divergencias entre los casos para lograr una mirada más amplia del tema investigado.

La investigación optó por un estudio de caso con propósito descriptivo (Yin, 2003) porque presenta cómo son las acciones sobre la evaluación del profesorado participante durante una unidad didáctica. A la vez, es instrumental (Stake, 2010), porque el análisis de cada 
docente con sus similitudes y diferencias, permitieron comprender una situación en particular y aportar conocimiento a la teoría, hasta ahora existente, en relación a las prácticas que tiene el profesorado sobre la evaluación, generando nuevas preguntas de investigación.

El límite en el estudio de caso puede estar dado por el número de participantes, un periodo de tiempo de observación, o la instancia de un problema o preocupación (ChaverraFernández, Gaviria-Cortés y González-Palacio, 2019), para esta investigación, el caso se limitó a una unidad didáctica en cada uno de los participantes.

\subsection{Categorías de análisis}

Analizar la acción evaluativa del profesorado en su globalidad es un asunto complejo, debido a las tramas y relaciones entre todos los aspectos. Es decir, no pueden entenderse los elementos que la componen (contenidos, actores, instrumentos y momentos) de manera aislada, sino en relación de unos con otros. Sin embargo, para efectos del análisis, se presentan en dos grandes categorías para facilitar la lectura, la primera son los instrumentos, actores y momentos y, la segunda, los contenidos evaluados.

\subsection{Selección de participantes}

La elección de los participantes fue intencional, lo que Goetz y LeCompte (1988) llaman "selección basada en criterios simple" (p. 98), por lo tanto, los profesores debían cumplir con las siguientes características: tener una titulación como licenciados en educación física, ser docentes activos de un colegio público, tener mínimo cinco años de experiencia en la docencia y especialmente, tener disposición y tiempo para participar en el estudio. En definitiva, participaron seis docentes, cuatro hombres y dos mujeres.

A continuación, se presenta un resumen con las características del profesorado participante en relación a la edad, años de experiencia docente, tema de la unidad didáctica, grupo observado y promedio de edad de los estudiantes de ese grado. Como parte de la confidencialidad, sus nombres han sido cambiados utilizando seudónimos para la presentación de los resultados.

Cuadro 1. Información del profesorado participante en la investigación.

\begin{tabular}{|c|c|c|c|c|}
\hline NOMBRE & $\begin{array}{l}\text { EDAD } \\
\text { (AÑOS) }\end{array}$ & $\begin{array}{c}\text { AÑOS } \\
\text { COMO } \\
\text { DOCENTE } \\
\end{array}$ & $\begin{array}{c}\text { TEMA DE LA UNIDAD } \\
\text { DIDÁCTICA }\end{array}$ & $\begin{array}{c}\text { GRADO } \\
\text { OBSERVADO/EDAD DE } \\
\text { LOS ESTUDIANTES } \\
\end{array}$ \\
\hline Pedro & 35 & 15 & Balonmano & Séptimo (12-13 años) \\
\hline Luis & 36 & 12 & Natación & Séptimo (12-13 años) \\
\hline María & 47 & 20 & Actividad física & Octavo (13-14 años) \\
\hline Diana & 41 & 17 & $\begin{array}{c}\text { Gimnasia con pequeños } \\
\text { elementos }\end{array}$ & Octavo (13-14 años) \\
\hline Carlos & 41 & 19 & Fútbol & Once (17-18 años) \\
\hline Juan & 32 & 11 & Recreación y contexto social & Once (17-18 años) \\
\hline
\end{tabular}

Fuente: Elaboración propia.

Entre las consideraciones éticas de la investigación se encuentran: El consentimiento informado, la confidencialidad de la información y la devolución de los hallazgos a los participantes. 


\subsection{Instrumentos de obtención de información}

La observación fue la principal estrategia de recolección de información. Se asumió el rol denominado observador (como) participante, que en palabras de Tójar (2006), "es aceptado por el grupo y participa con cierta distancia física y psicosocial” (p. 245). Este rol fue determinante para el logro de los objetivos del estudio, ya que las relaciones establecidas con el profesorado y el estudiantado permitieron un escenario de confianza y tranquilidad para realizar la observación, pero con una distancia prudente para analizar libremente las acciones que realizaban los docentes.

El uso de instrumentos para registrar la observación son en palabras de Sanmartín (2010) ante todo "una ayuda para fijar la memoria" (p. 162). En este sentido, los avances tecnológicos proporcionan ventajas técnicas que permiten registrar con precisión la información que surge en la investigación. Diversos autores han defendido el uso de recursos audiovisuales en la investigación (Del Rincón, Arnal, Latorre y Sans, 1995; Hammersley y Atkinson, 2001; Shrum, Duque y Brown, 2005). Es así que para este estudio se optó por utilizar una cámara de video digital.

Utilizar el video para registrar la fase interactiva de la enseñanza conlleva como principal beneficio la densidad de la información que proporciona (Bottorff, 2003). Es innegable que la imagen permite conocer aspectos que no se logran plasmar por otros medios de registro debido a la rapidez de las acciones.

Así mismo el video permite volver a retomar los acontecimientos tal y como sucedieron en cualquier momento del análisis, logrando captar detalles que no se consideraron importantes o se pasaron desapercibidos. Otra ventaja del video es que registra con fiabilidad las acciones y la expresión corporal del profesorado; este lenguaje no verbal constituye una fuente de información secundaria que apoya o va en contra de su lenguaje verbal y constituye un elemento clave para comprender el significado de las expresiones.

Otra estrategia de recolección de información fue el análisis documental. El propósito fue complementar, contrastar y validar la información de otras estrategias (Bowen, 2009; Fitzgerald, 2007; Massot, Dorio y Sabariego, 2012). Este análisis se realizó a los documentos oficiales como lo plantea Woods (1987) y fueron: las planillas que utiliza el profesorado para registrar las calificaciones y diferentes estrategias que utilizaron los docentes con los estudiantes para recolectar información: cuadernos fichas, trabajos escritos, exámenes.

\subsection{Trabajo de campo}

En el segundo momento de la investigación como lo denomina Galeano (2004), se inició el acceso al campo. Como lo dicen Hammersley y Atkinson (2001), acceder al campo no se limita a la presencia física "Es mucho más que una simple cuestión de conseguir o poseer un permiso para llevar a cabo la investigación” (p. 72). Esta relación investigadoraparticipantes debía sustentarse en la confianza, el respeto y el reconocimiento del otro como un interlocutor válido. El encuentro con los profesores en espacios fuera de la clase como las oficinas, compartir un café o algunos eventos académicos, permitieron intercambiar ideas, expectativas e inquietudes que fueron formando lazos de confianza y empatía que favorecieron el desarrollo del estudio. 
En los contactos iniciales con el profesorado se definieron los días y horas en que se realizarían las observaciones. Es necesario anotar que la investigación no definió con anterioridad las temáticas de las unidades didácticas, es decir, cada docente eligió libremente el grado y el tema con el cual quería participar.

El trabajo de campo se llevó a cabo en cada unidad didáctica elegida por los profesores, para un total de 51 clases grabadas. Esta observación sistemática permitió obtener una información detallada de los instrumentos/procedimientos que utilizó el profesorado para evaluar.

La salida del campo (Hammersley y Atkinson, 2001; Taylor y Bogdan, 1987) se dio al finalizar la unidad didáctica. Sin embargo, se continuó el contacto con el profesorado para obtener alguna información adicional y presentarles de manera individual los resultados del estudio.

\subsection{Análisis de la información}

El análisis de la información se asumió desde los planteamientos de Taylor y Bogdan (1987), los cuales proponen tres fases: fase de descubrimiento, fase de codificación y fase relativización de los datos. En la primera fase se realizó una lectura detallada de las fuentes de información y se identificaron las categorías iniciales.

En la fase de codificación se depuraron, expandieron o suprimieron las interpretaciones iniciales. La construcción de sentido en los datos se realizó de manera inductiva (Bonilla y Rodríguez, 1997), de tal forma que las categorías emergieron de la información con base en los patrones y recurrencias presentes en ellos. La información se codificó de acuerdo al tipo de fuente suministrada, el número que corresponde y el nombre de los docentes, ejemplo: C.2.M (Clase 2 de María); P.D (Planilla de seguimiento de Diana), T.E.5L (Texto del estudiante de Luis)

En la última fase de relativización de los datos (nombrada así por Deutsher y Mills, 1940, citados por Taylor y Bogdan 1987), se interpretó la información de acuerdo al contexto de cada participante, con el propósito de comprender la realidad educativa que vive cada docente y contrastarlo con los elementos teóricos abordados y el estado actual del objeto de estudio.

Para el tratamiento de la información se utilizó el programa informático Nvivo 10 para Mac (versión 11.3.2), lo que permitió hacer la refinación y depuración de la información que proponen Taylor y Bogdan (1987).

\section{Resultados y discusión}

\subsection{Instrumentos, actores y momentos}

Para iniciar, se presentan los instrumentos utilizados por el profesorado y su vínculo con los actores que intervinieron en su realización, así como los momentos en que se llevaron a cabo. En primer lugar, se encontró que la planilla de seguimiento es el instrumento principal que utiliza el profesorado para registrar, por medio de calificaciones, la información procedente de las observaciones no estructuradas de cada sesión. Estas planillas, son formatos que cada colegio define para registrar la evaluación, la asistencia, 
entre otros datos, pero no contienen ni reflejan criterios de evaluación que permitan realizar un seguimiento oportuno al aprendizaje.

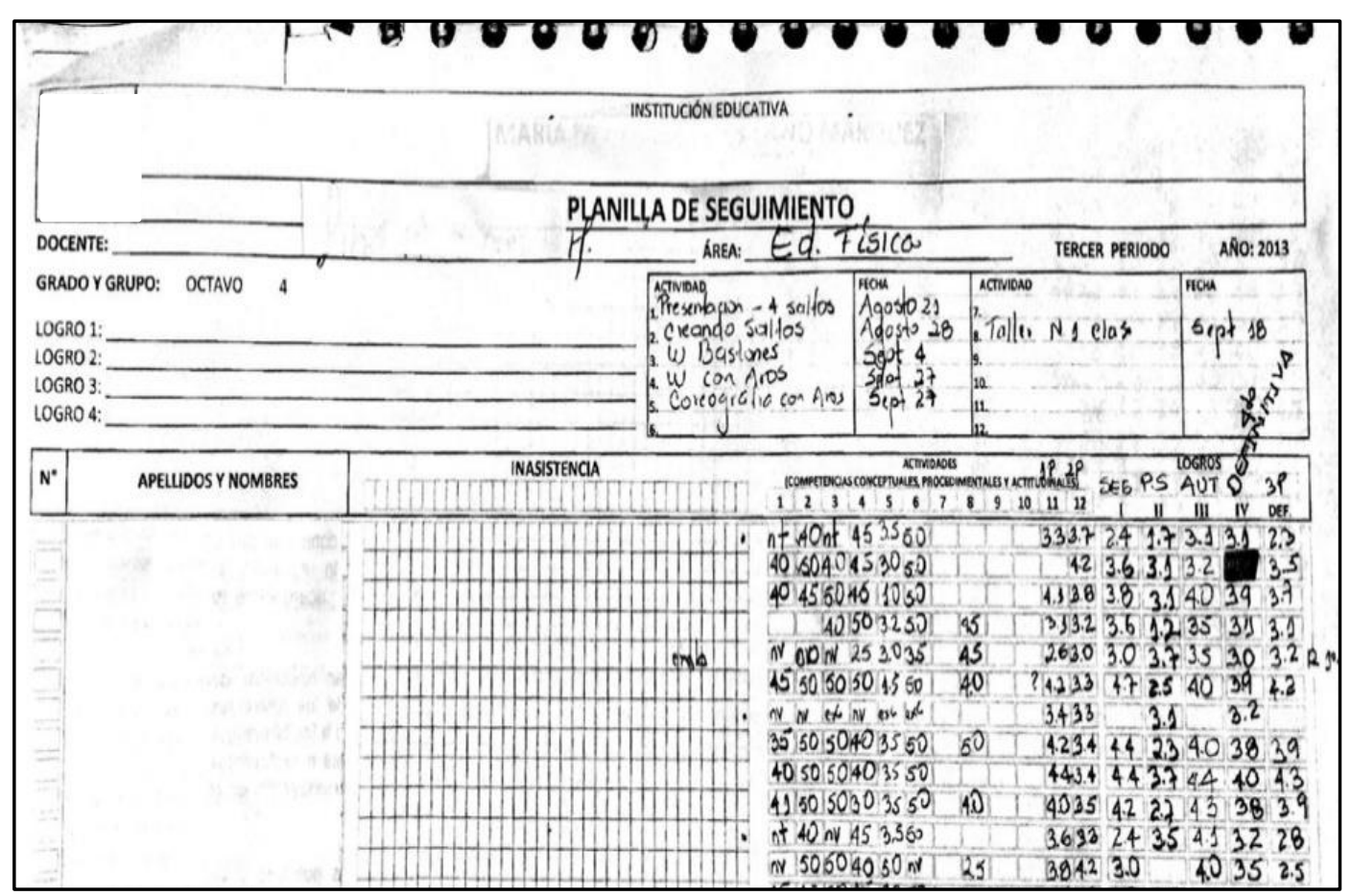

Figura 1. Planilla de seguimiento de Diana (P.D.)

Fuente: Profesora Diana participante del estudio.

El registro de información realizado en la planilla se basa en la observación libre del desempeño del estudiantado, convirtiéndose así la observación no estructurada en el procedimiento más relevante que utilizó el profesorado para evaluar. Esta práctica se corresponde con lo hallado por Sherer (2000) y Hein, Vieira y Mendes (2007), donde confirman que la observación asistemática y permanente es el medio más usual del profesorado para conseguir la información en la evaluación. Sin embargo, es necesario preguntarse ¿Qué es lo que se observa? ¿De dónde surge la calificación?, interrogantes que se abordarán más adelante en el apartado de contenidos.

Cabe mencionar, que en las planillas también quedaron consignadas otras calificaciones que surgieron de los trabajos teóricos, los exámenes, las autocalificaciones -cuando el estudiantado define su calificación total o parcial (López-Pastor, 2017) - y las coevaluaciones o evaluaciones entre pares. El registro en la planilla se convierte en una prueba fundamental ante el colegio, los padres de familia o el mismo estudiantado del proceso evaluativo realizado, de ahí que sus funciones están más cercanas a un instrumento de control que a una verdadera herramienta que permita recoger información para la evaluación del aprendizaje.

El uso de la planilla se convierte en el reflejo de la principal técnica de evaluación del profesorado, la heteroevaluación del aprendizaje, asumiendo por heteroevaluación la evaluación que tradicionalmente realiza el profesor sobre el estudiante (Uribe, 2009).

Si bien todos los docentes llevaron a cabo acciones de autoevaluación, coevaluación y especialmente autocalificación, el profesor es el actor principal de la evaluación durante 
las unidades didácticas observadas. La acción constante entre los profesores fue llamar a lista al finalizar la clase y emitir su calificación de acuerdo con criterios que no se han hecho explícitos, algunos con mayor frecuencia que otros, por ejemplo, Luis y Carlos, lo hicieron en seis de las siete clases observadas. El siguiente ejemplo, en la clase $n^{\circ} 8$ de Luis, ilustra ese procedimiento:

Duvan 3.0; Estefanía 4.5; Dahiana 4.0; Karen 4.0; Michel 4.0 ... (C.8.L.)

Este proceder del profesorado evidencia una calificación continua, que no es igual a una evaluación permanente en términos formativos, por el contrario, esta práctica está más vinculada a la evaluación sumativa, que como lo señalan Velázquez y Hernández (2004), pretende conocer los logros o resultados netos del aprendizaje. Sin embargo, como se verá más adelante, el criterio fundamental para emitir esta calificación no fue el aprendizaje en sí mismo sino la participación en la clase.

En las pocas ocasiones en que los profesores no calificaban, acudían a la autoevaluación. Esta participación del estudiantado se presentó de dos maneras: la autocalificación, entendida como la nota que se asigna el propio estudiante sin criterios concretos y la autoevaluación, en la que el estudiante se asigna una calificación, teniendo presente unos criterios establecidos. La primera opción fue la más recurrente, el profesor les preguntaba de manera individual o colectiva por una calificación.

\section{Los que hicieron el trabajo acá, ¿Qué nota creen que se merecen después del trabajo en clase? [5, responden todos]. (C.1.P.)}

En medio de la autocalificación, Diana y Pedro vieron la oportunidad de confrontar el juicio del estudiante con su propia opinión. Sin embargo, esta acción no llega a convertirse en una calificación dialogada en los términos de López et al., (2007), ya que finalmente no se llega a un consenso, sino que prevalece la nota del estudiante, aunque no se sustente con argumentos y, por tanto, carente de una función formativa.

Erick ¿Cuánto crees que te mereces hoy?, a conciencia, $[4,5] \ldots$ ¿ Eso crees que te mereces? Te lo puse, pero la próxima clase no voy a permitir la autoevaluación. (C.6.D.)

Es posible pensar que la falta de honestidad (o de objetividad) en el estudiantado en estos ejercicios, se deba a la poca costumbre de realizar actividades de autorregulación o reflexión, por tanto, ven en la autocalificación, la oportunidad de mejorar sus notas. A la vez, la falta de criterios evaluativos por parte del profesorado también permite que la autocalificación no sea sincera o ajustada a lo que espera el docente, dado que la pregunta principal hacia el estudiante fue: “Qué nota se merece?” y no se presentan elementos concretos que generen una reflexión en el estudiantado. Este tipo de preguntas, no promueven que el estudiante se interese en su propio aprendizaje (Trigueros-Cervantes, Rivera-García y De la Torre-Navarro, 2012), sino que refuerza la concepción de evaluación tradicional y sumativa. Como lo plantean Brown, Irving y Keegan (2008), la autoevaluación es un proceso complejo que se facilita cuando existen criterios claros y definidos al momento de realizarla, algo que no sucede en nuestro estudio.

La segunda forma de autoevaluación fue la que culminaba con una calificación en respuesta a preguntas o presentación de algunos criterios por parte del docente, para que, a partir de ahí, el estudiantado emitiera su calificación. Este tipo de autoevaluación fue muy escasa, sólo se presentaron cuatro autoevaluaciones de este tipo durante todas las clases (4 de 51). 
... ustedes van a poner la nota de cómo trabajaron hoy, escriben A: creatividad; B: Diálogo...

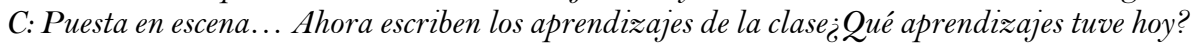
¿Qué dificultades? (C.2.J.)

Estas acciones del profesorado, dejan en evidencia la confusión terminológica y metodológica que tienen entre la autoevaluación y la calificación. Como lo plantea LópezPastor (2017), el peso de la tradición y el paso por el sistema escolar genera automatismos que tienden a que repitamos como docentes las practicas que se han vivido como estudiantes. Esta tradición sumativa de la evaluación, ejerce una gran influencia en el profesorado participante y les resulta difícil llevar a cabo acciones evaluativas que no estén ligadas a la calificación o el control en el aula.

Las calificaciones que se asignaba cada estudiante a sí mismo quedaban registradas en la planilla de seguimiento, pero las autoevaluaciones tuvieron un lugar especial en el cuaderno del estudiante. Este fue otro instrumento utilizado por el profesorado.

En general, el cuaderno del estudiante fue utilizado como un instrumento de registro, donde se plasmaron las tareas teóricas y las autoevaluaciones con una finalidad centrada en la calificación. Sólo el profesor Juan lo utilizó con una intención más amplia de reflexión sobre sus aprendizajes.

...vamos a sacar el cuaderno... ¿Qué dificultades tuve en la sesión o en la clase de hoy?...

¿Qué aprendizajes adquirí en la clase de hoy? (C.6.J.)

Para este profesor, el cuaderno también le permitió registrar la heteroevaluación de la enseñanza, es decir, la evaluación que puede realizar el estudiante hacia el profesor. Juan, por medio de preguntas, solicitó al grupo que emitiera un concepto sobre su desempeño docente y finalizara con una calificación. Este profesor fue el único, entre los participantes, que solicitó esta evaluación en dos oportunidades y no lo hizo al final de la unidad, sino en clases intermedias.

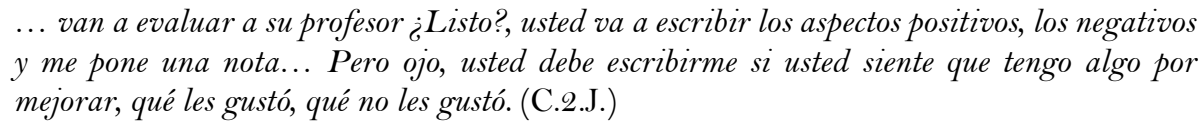

Esta heteroevaluación de la enseñanza, también la llevó a cabo Luis en la última sesión. De manera escrita le solicitó al estudiantado que lo calificaran (escala de 1 a 5 ) y justificaran su respuesta.

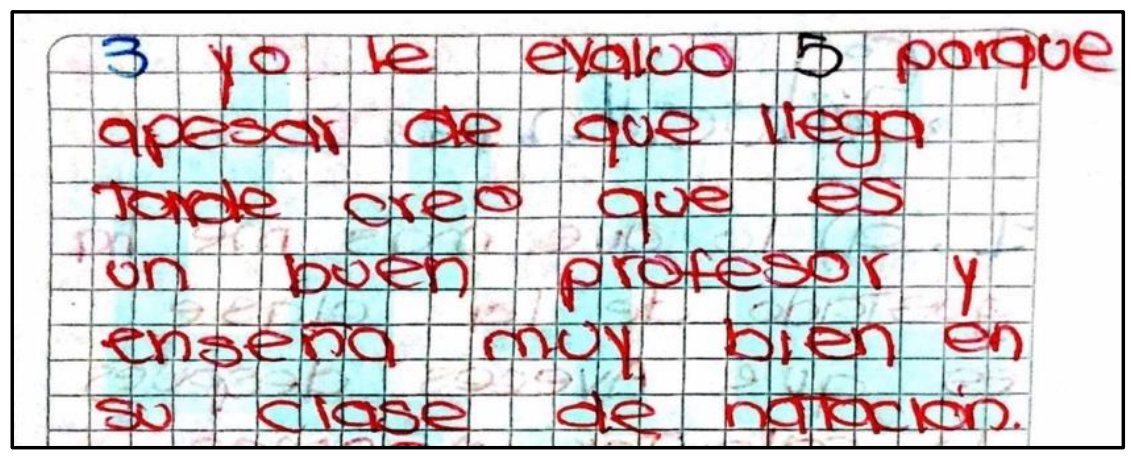

Figura 2. Evaluación de la enseñanza del estudiante 5 de Luis (T.E.5L.) Fuente: Profesor Luis participante del estudio. 
En el caso de María, la profesora realizó una pregunta durante toda la unidad didáctica que puede acercarse a una evaluación de su enseñanza. Al terminar la primera tarea en la clase número uno, le preguntó al estudiantado qué era lo que no les había gustado de la actividad.

Quién me dice qué no le gustó y por qué [Fue muy poquito tiempo], fue muy poquito tiempo... Qué más no les gustó [Profe los lazos muy corticos]. (C.2.M.)

Ante los comentarios realizados, la profesora presenta sus argumentos y justifica sus decisiones, pero no profundiza en las inquietudes del estudiantado. Por su parte los profesores Pedro y Carlos no realizaron ninguna actividad para evaluar su enseñanza.

La evaluación de la enseñanza puede presentarse en las tres fases que configuran la labor docente. Hernández y López (2004) proponen diversos criterios para realizar esta evaluación en cada fase, pero, lo encontrado en nuestra investigación, es que la evaluación de la enseñanza es escasa en la fase interactiva. Esta evaluación sólo se hizo presente cuando algunos profesores permitieron la participación del estudiantado, ya sea de manera escrita o verbal; pero, en general, la evaluación del profesorado se centra en el aprendizaje y desconoce la información que el estudiantado, los compañeros docentes, o ellos mismos desde la autoevaluación, pueden generar para mejorar su enseñanza.

Esta misma desatención a la evaluación de la enseñanza fue un hallazgo en un estudio anterior (Chaverra-Fernández, 2014), donde la inexperiencia, la poca motivación, la falta de tiempo o el desconocimiento de cómo realizar este proceso, son posibles razones para que el profesorado no realice una evaluación de su enseñanza desde todos los actores (auto, co y heteroevaluación).

Por otro lado, la coevaluación o evaluación del aprendizaje entre iguales (López-Pastor, 2017) no fue una estrategia muy frecuente entre el profesorado. Sólo Juan y Luis realizaron alguna actividad al respecto, con criterios y calificación al final.

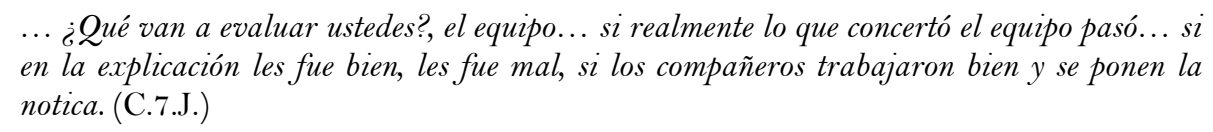

Otro instrumento común a Juan, Diana y Pedro fue la realización de la prueba teórica que solicita el colegio en cada periodo. Este examen se realiza al finalizar la unidad didáctica y es conocido por profesores y estudiantes como "pruebas saber", ya que las preguntas siguen el modelo de evaluación nacional colombiana que lleva el mismo nombre.

Para esta prueba, cada docente construye un examen y tiene un valor del $20 \%$ sobre el total de la calificación. Si bien es una obligación institucional, el profesorado lo asume como una oportunidad para identificar los saberes de los estudiantes y generar acciones de mejoramiento.

Vean muchachos ojo en las pruebas, yo no voy a preguntar nada raro de lo que ustedes han visto, ya saben que la recreación genera ¿Qué? [Placer] ¿Qué más? [Alegría, diversión], sí...hay aprendizaje, favorece el desarrollo humano ¿Qué más? Aprovechamiento de [el tiempo libre]... no voy a preguntar nada diferente en la prueba. (C.10.J.)

\subsection{Contenidos evaluados}

Carlos, Pedro, Diana y María, utilizaron talleres o trabajos teóricos para evaluar los contenidos conceptuales, todos calificables. 
... en la próxima clase les califico la cancha de balonmano y les califico las posiciones de cada jugador. (C.5.P.)

Adicional a los talleres escritos, Carlos, Pedro y Juan evaluaron constantemente estos contenidos por medio de preguntas, buscando que el estudiantado expresara sus ideas y saberes. En algunos momentos lo hicieron durante las tareas o al inicio de cada clase. Por ejemplo, Carlos, en una tarea de fútbol, para la actividad y les pregunta.

...paren ahí, acérquense un momento ¿Cuál es la estrategia que debemos hacer para lograr tumbar el cono? [Tirar para otro lado], ¿Cómo se llama eso? [Yo no sé], cambio de perfil, cambio de sentido de juego. ¿Qué hay que tener para cambiar de lado? [Silencio], lo deben tener los porteros, los volantes de creación ¿̨Cómo se llama eso?, [Visión] ¿Visión qué? [Visión periférica], bien, visión periférica. (C.5.C.)

Como señalan Velázquez, Hernández, Martínez y Martínez (2011), los contenidos de tipo conceptual se han ido incorporando a los currículos de educación física en diferentes países con la intención, entre otros aspectos, de lograr el desarrollo de una mayor autonomía y la adopción de estilos de vida activos del estudiantado.

Por su parte, los contenidos actitudinales fueron tal vez los más evidentes en la evaluación del profesorado, especialmente al momento de calificar. Por un lado, los criterios propuestos para realizar las autoevaluaciones y/o calificaciones, estuvieron centrados en reflexionar sobre la participación, la responsabilidad, la actitud y el comportamiento en la clase.

... esta vez me van a dar la nota ustedes, de acuerdo al comportamiento que tuvieron en la
clase... la nota es independientemente si sabe hacer el salto o no lo sabe hacer. (C.1.D.)

Por otro lado, el comportamiento y la participación fueron los criterios determinantes que tuvo el profesorado para emitir la calificación al finalizar las clases. Quienes participaban activamente de las tareas y no tenían un comportamiento sancionable, obtenían una calificación positiva; por el contrario, el estudiantado que no llevaba el uniforme, utilizaba indebidamente los materiales, agredía a sus compañeros o simplemente no participaba de la clase sin una justificación, obtenía una mala calificación.

En definitiva, cabe afirmar que el profesorado centró su evaluación en aspectos disciplinarios, pero no se rescatan actitudes como el trabajo en equipo, la disposición para realizar las tareas, el compañerismo o la autonomía. El marcado interés por evaluar las actitudes se corresponde con lo encontrado por Foglia (2014), quien asegura que, entre el profesorado, el componente actitudinal recibe una atención especial, al punto en que la mayoría reconoce que "el área se pierde especialmente por situaciones de tipo actitudinal, cuando los estudiantes son irresponsables e indisciplinados” (p. 113).

Asimismo, el estudio de Hein et al. (2007), concluyó que el profesorado centraba su evaluación en la dimensión comportamental y en la participación en las clases, dejando de lado otros contenidos. En esta misma línea, los estudios de Sherer (2000) y ChaverraFernández y Hernández-Álvarez (2019) encontraron que, las actitudes del estudiantado durante las clases y la frecuencia de participación, son el principal referente del profesorado para evaluar, dado que no hay una planificación de la evaluación o definición de criterios.

En nuestra investigación se encontró que la evaluación de las actitudes es una acción informal, basada sólo en la observación del comportamiento que tuvo el estudiantado durante la clase, una evaluación momentánea, pero carente de criterios e instrumentos que 
permitan hacer un seguimiento a lo largo de las clases. En ese sentido, el profesorado estudiado cae en lo que Prat (2003) señala como criterios subjetivos y poco sistemáticos para evaluar las actitudes. Resultados similares a los de nuestro estudio encontró Vargas (2012), cuando concluye que el profesorado no asumía la evaluación de las actitudes como un proceso sistemático, sino aislado y poco consciente.

La utilización de la planilla de seguimiento para calificar cada sesión es una muestra de la falta de criterios en el profesorado para evaluar las actitudes. Esta planilla no arroja una información importante que les permita proponer acciones formativas de cambio o mejora durante el desarrollo de la unidad didáctica, sólo favorece registrar los procesos disciplinarios que cada institución tiene definidos.

En esta evaluación basada en la observación libre que llevan a cabo los participantes en el estudio, no era un criterio relevante el desempeño del estudiantado. Es decir, ningún profesor se refirió a que su calificación tuviera en cuenta el aprendizaje o no de las tareas motrices. En este sentido, es difícil identificar en las acciones del profesorado la manera en que los procedimientos fueron evaluados, dado que no se observaron acciones o instrumentos que permitieran identificar que el desempeño del estudiantado fuera motivo de evaluación.

En síntesis, como también señalan diferentes autores, la falta de claridad en los criterios de evaluación y el poco interés por evaluar el desarrollo motor, conllevan a que el profesorado centre la mirada en elementos poco educativos y subjetivos como el uniforme, el esfuerzo, la asistencia o la conducta (López-Pastor, Kirk, Lorente-Catalán, MacPhail, y Macdonald, 2013; Siedentop, 2008), estando, por tanto, ausente del proceso una verdadera opción de evaluación de carácter formativo tanto en los aspectos del aprendizaje como de la propia enseñanza.

\section{Conclusiones}

En términos generales, el análisis de la acción evaluativa del profesorado permite concluir que no se evidencia una recolección de información sistemática y oportuna, que se valga de instrumentos precisos, con criterios concretos que permitan identificar cómo avanza el aprendizaje y la enseñanza para proponer acciones de mejora, finalidad fundamental de una evaluación formativa.

La acción evaluativa demuestra la marcada importancia que le otorga el profesorado a la calificación, convirtiéndose en la máxima expresión de su evaluación. De esta manera, la evaluación ligada a la calificación, permea todos los elementos que la constituyen, es decir, los actores, momentos, instrumentos y contenidos. En el caso de los actores, el protagonista principal es el docente, asumiendo casi toda la responsabilidad de calificar el aprendizaje y dejando pocas opciones al estudiantado de participar a través de la autoevaluación, coevaluación y heteroevaluación de la enseñanza.

Asimismo, los momentos de la evaluación se asumen como una calificación continua, ligada a la evaluación sumativa en la que cada nota es determinante para la evaluación final.

En el caso de los instrumentos, se identificó la planilla de seguimiento como el instrumento privilegiado para registrar las calificaciones y controlar la participación del 
estudiantado. En consecuencia, el profesorado carece de instrumentos con criterios definidos para obtener información valiosa que permita realizar ajustes al aprendizaje o la enseñanza.

En cuanto a los contenidos, se evidenció que el profesorado centra su atención en elementos disciplinarios y de forma (llevar uniforme o no, participar, portarse bien, etc.), disipando una acción más decidida sobre la educación en valores y actitudes más vinculadas al desarrollo personal y social del estudiantado (autonomía, trabajo en equipo, solidaridad). De igual manera, los contenidos procedimentales son los más ausentes en la evaluación del aprendizaje, no pudiéndose observar cómo fueron evaluados, ni identificar su contribución en la calificación que se asignaba al final de cada clase. Dado el carácter de la propia asignatura (educación física), la carencia de una verdadera evaluación formativa hacia los contenidos procedimentales, constituye una relevante laguna en el marco de una educación que atienda a las diferentes potencialidades de la persona.

Se hace necesario continuar con la investigación sobre la acción evaluativa tratando de hacer visibles en contextos reales sus características, fortalezas y debilidades, especialmente estas últimas, para transitar desde los juicios de valor hacia la elaboración de propuestas de una evaluación que se oriente a la mejora de la calidad del proceso de enseñanza y aprendizaje en educación física.

\section{Referencias}

Barrientos, E. y López-Pastor, V. M. (2017). Evaluación orientada al aprendizaje en educación superior: últimas tendencias. Infancia, Educación y Aprendizaje, 3(2), 582-587.

Benevides, M. y Santos, C. (2004). Avaliação em Educação Física Escolar: o professor como sujeito avaliado no processo educativo. Diálogos Possíveis, 3(2), 159-174.

Bonilla, E. y Rodríguez, P. (1997). Más allá del dilema de los métodos: la investigación en ciencias sociales. Bogotá: Norma.

Bottorff, J. (2003). El uso de las grabaciones de vídeo en la investigación cualitativa. En J. Morse (Ed.), Asuntos críticos en los métodos de investigación cualitativa (pp. 284-304). Medellín: Universidad de Antioquia.

Bowen, G. (2009). Document analysis as a qualitative research method. Qualitative Research Journal, 9(2), 27-40. https://doi.org/10.3316/QRJ0902027

Brown, G., Irving, E. y Keegan, P. (2008). An introduction to educational assessment, measurement, and evaluation: improving the quality of teacher-based assessment (2a ed.). Nueva Zelanda: Pearson Education New Zealand.

Cabezas, D., González, C. y Carpintero, E. (2009). Evaluar la evaluación: cuestionario sobre prácticas de evaluación. EduPsykhé: Revista de Psicología y Educación, 8(1), 51-61.

Carless, D. (2007). Learning-oriented assessment: conceptual bases and practical implications. Innovations in Education and Teaching International, 44(1), 57-66. https://doi.org/10.1080/14703290601081332

Cerda, H. (2000). La evaluación como experiencia total. Logros-objetivos-procesos- competencias y desempeño. Bogotá: Cooperativa Editorial Magisterio.

Chaverra-Fernández, B. (2014). Significados otorgados a la evaluación de la enseñanza y el aprendizaje: Interpretación a partir de un grupo de maestros de educación física. Estudios 
pedagógicos (Valdivia), 40(2), 65-82. https://doi.org/http://dx.doi.org/10.4067/So71807052014000300004

Chaverra-Fernández, B. (2017a). Pensamiento y acción evaluativa del profesorado de educación física de educación secundaria y media: Estudio de casos en Medellín-Colombia. Tesis doctoral no publicada. Universidad Autónoma de Madrid, Madrid.

Chaverra-Fernández, B. (2017b). Análisis del discurso evaluativo. Infancia, Educación y Aprendizaje, 3(2), 723-728. https://doi.org/http://dx.doi.org/10.22370/ieya.2017.3.2.808

Chaverra-Fernández, B. y Hernández-Álvarez, J. L. (2019). La planificación de la evaluación en educación física: Estudio de casos sobre un proceso desatendido en la enseñanza. Revista Electrónica Educare, 23(1), 1-21. https://doi.org/10.15359/ree.23-1.12

Chaverra-Fernández, B., Gaviria-Cortés, D. y González-Palacio, E. (2019). El estudio de caso como alternativa metodológica en la investigación en educación física, deporte y actividad física. Conceptualización y aplicación. Retos. Nuevas tendencias en Educación Fisica, Deporte y Recreación, $1^{\circ}$ semestre(35), 371-377.

Clark, C. y Peterson, P. (1990). Procesos de pensamiento de los docentes. En M. Wittrock (Ed.), La investigación de la enseñanza III (pp. 442-539). Barcelona: Paidós.

Conte, L. y Moreno, J. (2000). La autoevaluación como elemento de reflexión y formación en Educación Física. En O. Contreras (Ed.), La formación inicial y permanente del profesor de Educación Física. Volumen II (pp. 27-37). Cuenca: Universidad de Castilla-La Mancha.

Del Rincón, D., Arnal, J., Latorre, A. y Sans, A. (1995). Técnicas de investigación en ciencias sociales. Madrid: Dykinson.

Del Villar, F., Ramos, L., Cervelló, E., Julián, J., y Jiménez, R. (2002). El análisis temático del pensamiento reflexivo del profesor de educación física en su formación inicial. Revista Internacional de Ciencias Sociales y Humanidades, 12(2), 45-64.

Denzin, N. y Lincoln, Y. (2005). Introduction: the discipline and practice of qualitative research. En N. Denzin y Y. Lincoln (Eds.), Handbook of Qualitative Research (3a , pp. 1-32). Thousand Oaks, CA: Sage.

Fernández, J. (2007). Estudio del pensamiento del profesor de Educación Física en las etapas de primaria y secundaria. Tesis doctoral no publicada, Universidad Complutense de Madrid, Madrid.

Fitzgerald, T. (2007). Documents and documentary analysis: reading between the lines. En A. Briggs y M. Coleman (Eds.), Research methods in educational leadership and management ( pp. 278-294). Londres: Sage Publications.

Foglia, G. (2014). Evaluación en educación física: concepciones y prácticas docentes. Voces y Silencios: Revista Latinoamericana de Educación, 5(2), 99-117.

Galeano, M. (2004). Estrategias de investigación social cualitativa. El giro de la mirada. Medellín: La Carreta.

García, H. (2003). Antecedentes, evolución y aportaciones de la investigación del pensamiento y conocimiento del profesor de educación física. Revista de Estudios e Experiencias Educativas, 19, 105-133.

Giné, N., Parcerisa, A., Llena, A., París, E. y Quinquer, D. (2003). Planificación y análisis de la práctica educativa. La secuencia formativa: fundamentos y aplicación. Barcelona: Graó.

Giraldo, L., Rubio, E. y Fernández, J. (2009). Caracterización del discurso pedagógico del docente de educación física e identificación de los actos de habla que estimulan la creatividad motriz. Ágora para la EF y el Deporte, 11, 25-41. 
Goetz, J. y LeCompte, M. (1988). Etnografía y diseño cualitativo en investigación educativa. Madrid: Morata.

Hall, T., y Smith, M. (2006). Teacher Planning, Instruction and Reflection: What We Know About Teacher Cognitive Processes. Quest, 58(4), 424-442. https://doi.org/10.1080/00336297.2006.10491892

Hammersley, M. y Atkinson, P. (2001). Etnografía. Métodos de investigación. Barcelona: Paidós.

Hein, E., Vieira, J. y Mendes, J. (2007). Metamorfoses na avaliação em Educação Física: da formação inicial à prática pedagógica escolar. Movimento, 13(2), 55-76. https://doi.org/10.22456/1982-8918.3546

Hernández Álvarez, J. L. y López Rodríguez, A. (2004). Evaluación de la enseñanza: análisis y propuestas. En J.L. Hernández y R. Velásquez (Eds.), La evaluación en educación física. Investigación y práctica en el ámbito escolar (pp. 49-76). Barcelona: Graó.

Jackson, P. (1992). La vida en las aulas. Madrid: Morata.

Jinhong, J. (2012). The focus, role, and meaning of experienced teachers' reflection in physical education. Physical Education and Sport Pedagogy, 17(2), 157-175. https://doi.org/10.1080/17408989.2011.565471

López-Pastor, V. (2005). La participación del alumnado en la evaluación: la autoevaluación, la coevaluación y la evaluación compartida. Tándem: Didáctica de la Educación Física, 17.

López-Pastor, V. (2017). Evaluación formativa y compartida: evaluar para aprender y la implicación del alumnado en los procesos de evaluación y aprendizaje. En V. López-Pastor y Á. PérezPueyo (Eds.), Evaluación formativa y compartida en educación: experiencias de éxito en todas las etapas educativas (pp. 34-68). León: Universidad de León.

López-Pastor, V., Kirk, D., Lorente-Catalán, E., MacPhail, A. y Macdonald, D. (2013). Alternative assessment in physical education: a review of international literature. Sport, Education and Society, 18(1), 57-76. https://doi.org/10.1080/13573322.2012.713860

López-Pastor, V., Barba-Martín, J., Monjas-Aguado, R., Manrique-Arribas, J., Heras-Bernardino, C., González-Pascual, M. y Gómez-García, J. (2007). Trece años de evaluación compartida en educación fisica. Revista Internacional de Medicina y Ciencias de la Actividad Física y el Deporte, 7(26), 69-86.

López-Rodríguez, A. (2012). La calidad de los procesos de comunicación en el aula de Educación Física: un estudio sobre calidad del discurso docente en profesorado de la Comunidad de Madrid. Tesis doctoral no publicada, Universidad Autónoma de Madrid, Madrid.

Massot, I., Dorio, I., y Sabariego, M. (2012). Estrategias de recogida de información y análisis de la información. En R. Bisquerra (Ed.), Metodología de la investigación educativa (pp. 329-366). Madrid: La Muralla.

Ní Chróinín, D. y Cosgrave, C. (2013). Implementing formative assessment in primary physical education: teacher perspectives and experiences. Physical Education and Sport Pedagogy, 18(2), 219-233. https://doi.org/10.1080/17408989.2012.666787

Nunziati, G. (1990). Pour construire un dispositif d'évaluation formatrice. Cahiers pédagogiques, 280, $47-64$.

Pérez, A. y Gimeno, J. (1988). Pensamiento y acción del profesor: de los estudios sobre la planificación al pensamiento práctico. Infancia y Aprendizaje, 42(42), 37-63. https://doi.org/10.1080/02 103702.1988.10822201 
Pradas, R. (2010). El estudio del pensamiento del profesorado sobre la toma de decisiones interactivas: análisis de un caso en educación física escolar. Cultura y Educación, 22(1), 2136. https://doi.org/10.1174/113564010790935187

Prat, M. (2003). Los contenidos actitudinales: las actitudes, los valores y las normas. En M. Prat y S. Soler (Eds.), Actitudes, valores y normas en la educación física y el deporte. Reflexiones y propuestas didácticas (pp. 21-33). Barcelona: Inde.

Rosales, C. (2000). Evaluar es reflexionar sobre la enseñanza. Madrid: Narcea.

Sanmartín, R. (2010). La observación participante. En M. García, J. Ibáñez, y F. Alvira (Eds.), El análisis de la realidad social. Métodos y técnicas de investigación (pp. 145-165). Madrid: Alianza.

Sherer, A. (2000). O conhecimento pedagógico do professor de educaçao física na escola pública da rede estadual de ensino e sua relação com a prática docente. Tesis doctoral ni publicada, Universidade Federal do Rio Grande do Sul.

Shön, D. (1998). El Profesional reflexivo: cómo piensan los profesionales cuando actúan. Barcelona: Paidós.

Shrum, W., Duque, R. y Brown, T. (2005). Digital Video as research practice: Methodology for the millennium. Journal of Research Practice, 1(1), M4.

Siedentop, D. (2008). Aprender a enseñar la educación física. Barcelona: Inde.

Stake, R. (2010). Investigación con estudio de casos. Madrid: Morata.

Taylor, S. y Bogdan, R. (1987). Introducción a los métodos cualitativos de investigación. Barcelona: Paidós.

Tójar, J. C. (2006). Investigación cualitativa. Comprender y actuar. Madrid: La Muralla.

Tójar, J. C. y Mena, E. (2011). Innovaciones educativas en el contexto andaluz. Análisis multicaso de experiencias en Educación Infantil y en Educación Primaria. Revista de Educación, 354(Enero-Abril), 499-527.

Trigueros-Cervantes, C., Rivera-García, E. y De la Torre-Navarro, E. (2012). La evaluación en el aula universitaria: del examen tradicional a la autoevaluación. Revista Internacional de Medicina y Ciencias de la Actividad Fisica y del Deporte, 12 (47), 473-491.

Tristán, J. (2010). La relación de la planificación en la interacción sobre los comportamientos del profesorado principiante y con experiencia así como la de sus alumnos en un estudio de casos. Tesis doctoral no publicada, Universidad de Murcia, Murcia.

Ureña, N., Ureña, F., Velandrino, A. y Alarcón, F. (2006). Resultados de un caso práctico de autoevaluación en educación física en la etapa de primaria. Apunts: Educación Física y Deportes, 86(4 trimestre), 22-33.

Uribe, I. (2009). La evaluación en la educación física. En I. Uribe y D. Gaviria (Eds.), Guía Curricular para la Educación Física (pp. 165-170). Medellín: Universidad de Antioquia.

Vargas, A. (2012). Creencias del profesorado de Educación Física con respecto a la evaluación de actitudes: un estudio de caso. Trabajo de fin de Máster. Máster Universitario en innovación, evaluación y calidad de la educación física, Universidad Autónoma de Madrid, Madrid.

Velázquez-Buendía, R., Hernández-Álvarez, J. L, Martínez-Gorroño, M. E. y Martínez de Haro, V. (2011). Educación Física y conocimiento téorico-conceptual: estudio trans-cultural en Argentina, Brasil y España. Revista de Educación, 356, 653-675. https://doi.org/10-4438/1988-592X-RE-2010-356-055 
Velázquez, R. y Hernández, J. (2004). Evaluación en educación y evaluación del aprendizaje en educación física. En J. Hernández y R. Velázquez (Eds.), La evaluación en educación física. Investigación y práctica en el ámbito escolar (pp. 11-47). Barcelona: Graó.

Velázquez, R., Hernández, J., Garoz, I., López, C., López, M., Maldonado, A. y Castejón, J. (2007). Calidad de enseñanza en Educación Física y Deportiva y discurso docente: el caso de la Comunidad de Madrid. Revista de Educación, 344, 237-238.

Woods, P. (1987). La escuela por dentro. Etnografía en la investigación cualitativa. Madrid: Paidós.

Yin, R. (2003). Case study research: Design and methods. Thousand Oaks, CA: Sage.

Zeichner, K. (1993). El maestro como profesional reflexivo. Cuadernos de Pedagogía, 220, 44-49.

\section{Cv de los autores}

\section{Beatriz Elena Chaverra Fernández}

Licenciada en Educación Física, Magister en Educación y Desarrollo Humano, Doctora en Ciencias de la Actividad Física y el Deporte de la Universidad Autónoma de Madrid (España), profesora asociada del Instituto de Educación Física de la Universidad de Antioquia, integrante del grupo de investigación Cultura Somática, participante como investigadora principal y coinvestigadora en diversos proyectos de investigación educativos. Miembro de la Red de Evaluación Formativa y Compartida en Educación. ORCID ID: 0000-0002-1183-8488. Email: beatriz.chaverra@udea.edu.co

\section{Juan Luis Hernández Álvarez}

Licenciado en Educación Física (1982) y Doctor en Filosofía y Ciencias de la Educación (1992), desarrolla su labor docente y de investigación como Catedrático en la Universidad Autónoma de Madrid. Antes de su desempeño profesional en la Universidad, ha sido Profesor de Educación Física en los niveles de Educación Primaria y Secundaria durante 17 años. Ha publicado un número relevante de trabajos en diferentes formatos: 12 libros, 25 capítulos de libros, más de 55 artículos en revistas especializadas, así como un amplio número de publicaciones en actas de congresos. Ha dirigido numerosos trabajos de investigación, destacando su papel de Investigador principal Responsable en diferentes proyectos competivos nacionales e internacionales. Es miembro de consejos científicos o asesores de diversas revistas, así como revisor de publicaciones. ORCID ID: 0000-00022327-0076. Email: juanluis.hernandez@uam.es 